\title{
Influence du polyéthylène oxyde (PEO) de différentes masses moléculaires sur les propriétés rhéologiques des suspensions d'argile
}

\author{
Koblan Wilfried EBAGNININ ${ }^{1 *}$, Adel BENCHABANE ${ }^{2}$ et Karim BEKKOUR \\ ${ }^{1}$ URF Sciences et Gestion de l'Environnement, Université d'Abobo-adjamé 2 B.P. 801 Abidjan 02, Côte \\ d'Ivoire. \\ ${ }^{2}$ Département de Génie Mécanique, Université Mohamed Khider BiskraB.P. 145 R.P. 07000, Biskra, Algeria. \\ ${ }^{3}$ Institut de Mécanique des Fluides et des Solides UMR CNRS-Université Louis Pasteur 7507 2, rue \\ Boussingault 67000 Strasbourg, France. \\ *Auteur correspondant, E-mail: koblanwill@yahoo.fr; Tel.: +225-49594561
}

\section{RESUME}

Le but de ce travail est d'étudier l'effet du polyéthylène oxyde (PEO) (polymère non ionique), utilisé généralement dans la formulation des boues de forage, sur le comportement rhéologique de suspensions de bentonite. Des mesures rhéologiques, granulométriques et zétamétriques ont été effectuées sur une suspension de bentonite, des solutions de PEO (polyéthylène oxyde de masse moléculaire de $4.10^{6}, 10^{6}, 4.10^{5}$ et $10^{5}$ g.mol ${ }^{-}$ ${ }^{1}$ ) et des mélanges bentonite-PEO à différentes concentrations de polymères. L'étude a révélé une forte augmentation (d'autant plus importante que la concentration et la masse moléculaire du PEO augmentent) de la viscosité, de la contrainte seuil et des propriétés viscoélastiques de la suspension argileuse. L'origine de ce changement de comportement a été discutée sur la base de l'étude zétamétrique et granulométrique. Cette dernière a mis en évidence des liaisons pont assurées par les chaînes de polymères entre les particules de bentonite.

(c) 2012 International Formulae Group. All rights reserved.

Mots clés : Boues de forage, bentonite, polyéthylène oxyde.

\section{INTRODUCTION}

Les systèmes bentonite-polymères sont couramment utilisés dans de nombreuses applications industrielles, notamment dans la formulation des fluides de forage. Les propriétés de ces boues argileuses à base d'eau sont largement modifiées par la présence de polymères, de tensioactifs ou d'électrolytes. Dans la littérature, on trouve de nombreux travaux traitant les systèmes "eauargile-additif" où l'additif est souvent un polymère non ionique. En s'intéressant aux interactions particule-additifs, les auteurs couplent souvent des mesures rhéologiques à des techniques physicochimiques (Diffraction par rayons X, spectrométrie infrarouge, etc.,) (Rossi et al., 2006a,b ; Alemdar et al., 2005a), afin de mieux élucider les interactions qui puissent exister entre la particule et les additifs. Des travaux antérieurs menés à l'Institut de Mécanique des Fluides et des Solides de Strasbourg (France) (IMFS) ont permis d'établir une synthèse bibliographique sur la microstructure et la caractérisation colloïdale et rhéologique des suspensions de bentonite sans et avec l'ajout de 
polymères/tensioactifs (Benchabane, 2006 ; Benchabane et Bekkour, 2006). Des mesures rhéologiques, granulométriques et de diffraction par rayons $\mathrm{X}$, ont permis d'approfondir la compréhension du caractère rhéologique des mélanges eau-bentonitepolymères anioniques (CMC, SDS, xanthane) et la nature des interactions particule-particule et particule-additif anionique (Benchabane, 2006 ; Benchabane et Bekkour, 2006).

Dans cet article, l'additif utilisé est le polyéthylène oxyde (PEO), un polymère non ionique de chaîne linéaire $-[\mathrm{CH} 2-\mathrm{CH} 2-\mathrm{O}]_{n}-$, souvent utilisé dans la formulation des fluides de forage. Une étude antérieure (Ebagninin et al., 2009) a permis de mettre en évidence le comportement rhéologique du polyéthylène oxyde (PEO).

Le but principal de cet article est d'une part d'étudier le comportement rhéologique des solutions de polyéthylène oxyde (PEO) (polymère non ionique) et d'autre part de connaître l'effet de ce même polymère non ionique (de différentes masses moléculaires) sur le comportement rhéologique de suspension de bentonite (suspension de base).

\section{MATERIEL ET METHODES Matériaux et appareillages}

L'argile utilisée est une bentonite commerciale (VWR Prolabo). Le polymère, le polyéthylène oxyde (PEO), est hydrosoluble, de différentes masses moléculaires : $10^{5}, 4.10^{5}$ $10^{6}$ et $4.10^{6}$ g.mol ${ }^{-1}$.

Les propriétés rhéologiques des suspensions ont été mesurées à une température maintenue constante à $20{ }^{\circ} \mathrm{C}$ à l'aide d'un rhéomètre à contrainte imposée (AR 2000). Les géométries de mesure choisies sont : $i$. plan-cône $\left(6 \mathrm{~cm}, 1^{\circ}\right)$ pour les solutions de polymères, ii. plan-cône $(6 \mathrm{~cm}, 2$ $\left.{ }^{\circ}\right)$ pour les suspensions de bentonite et les mélanges bentonite-PEO de faibles masses moléculaires $\left(10^{5}\right.$ g. $\left.\mathrm{mol}^{-1}\right)$ et iii. plan-plan (6 $\mathrm{cm}$ ), pour les mélanges de masses moléculaires élevées. Afin d'éviter les problèmes d'évaporation au cours des manipulations, le dispositif de mesure a été placé dans une ambiance saturée en vapeur d'eau.

\section{Mode préparatoire et méthodes de mesure}

Pour étudier l'influence de l'ajout du PEO sur la rhéologie du système bentoniteeau, une suspension de bentonite (sans additif), des solutions de PEO et des mélanges bentonite-PEO à différentes concentrations et de différentes masses moléculaires ont été préparés.

La préparation des solutions de PEO a été réalisée en utilisant de l'eau déminéralisée à température ambiante. Le polymère est dissout sous agitation magnétique durant 48 heures.

Tous les rhéogrammes ont été obtenus en suivant une même rampe montante de contrainte, relativement lente, choisie en respectant le temps de relaxation du matériau $(0,033 \mathrm{~Pa} / \mathrm{s})$.

Pour mieux comprendre la structure interne des solutions de polymère, des mesures rhéologiques ont été effectuées (en régime dynamique). Il s'agit de mesurer : $i$ ) le module élastique $\left(G^{\prime}\right)$ (qui est proportionnel à l'énergie élastique emmagasinée et restituée au cours d'une période) et, ii) le module visqueux $\left(G^{\prime \prime}\right)$ (l'énergie dissipée par frottement visqueux au cours du même cycle). Ces grandeurs rhéologiques ( $G^{\prime}$ et $G^{\prime \prime}$ ) dépendent de la nature du matériau et de la température mais également de la fréquence $(\omega)$ à laquelle le matériau est sollicité au cours des essais dynamiques.

Par conséquent, un échantillon peut avoir un comportement très visqueux à une fréquence donnée et très élastique à une autre. Pour nos solutions de polymère, nous avons effectué des mesures sur une gamme de fréquences allant de $10^{-2}$ à $10 \mathrm{~Hz}$. Les contraintes appliquées varient de $2.10^{-3}$ à $2.10^{-}$ ${ }^{2} \mathrm{~Pa}$ pour les solutions de PEO de masses moléculaires $\left(10^{5}\right.$ et $\left.4 \cdot 10^{5} \mathrm{~g} \cdot \mathrm{mol}^{-1}\right)$, de $2 \cdot 10^{-2}$ à $0.5 \mathrm{~Pa}$ pour les solutions de PEO de masse moléculaire $\left(10^{6}\right.$ g.mol $\left.{ }^{-1}\right)$ et de $2.10^{-2}$ à $1 \mathrm{~Pa}$ pour les solutions de PEO $\left(4.10^{6} \mathrm{~g} \cdot \mathrm{mol}^{-1}\right)$. Toutes ces contraintes appliquées sont 
suffisamment faibles pour rester dans le domaine de viscoélasticité linéaire.

Le mode préparatoire des suspensions à base d'argile à été établi dans des études antérieures pour obtenir des résultats reproductibles (Benchabane, 2006 ; Ebagninin et al., 2006a, 2009). Une suspension de bentonite sans additif (suspension de référence ou de base) est préparée par dispersion de la poudre de bentonite dans de l'eau distillée à une concentration de 5\%. L'homogénéisation est obtenue par agitation magnétique en continu pendant 4 heures puis la suspension est laissée au repos pendant 24 heures pour obtenir un bon gonflement de l'argile.

La préparation des mélanges eaubentonite-polymères consiste à ajouter progressivement le $\mathrm{PEO}$, à la concentration voulue, dans la suspension de bentonite de base. Le mélange est agité par la suite en continu pendant 24 heures à l'aide d'un agitateur magnétique. Ainsi, toutes les mesures présentées dans cet article sont réalisées sur des mélanges âgés de 7 à 10 jours avec une histoire mécanique d'une heure d'agitation quotidienne après les 24 heures d'homogénéisation.

Tous les rhéogrammes (suspensions de base et les mélanges) ont été obtenus en suivant toutes les conditions de précisaillement définies (application d'un précisaillement de $10 \mathrm{~s}^{-1}$ durant 2 minutes, suivi d'un repos de 2 minutes) et la même rampe montante de contrainte $\left(0,033 \mathrm{~Pa}^{-1}{ }^{-1}\right)$.

Pour effectuer les essais viscoélastiques, une contrainte de 0,018 $\mathrm{Pa}$ a été appliquée pour la bentonite de base. Pour tous les mélanges, les contraintes appliquées avaient des valeurs comprises entre 0,018 et 3 Pa. Avant chaque test, on applique un précisaillement de $10 \mathrm{~s}^{-1}$ durant 2 minutes, suivi d'un repos de 2 minutes sur l'échantillon.

Des essais préliminaires ont permis de s'assurer que ces contraintes sont bien dans la limite du domaine de viscoélasticité linéaire.

Les mesures dynamiques ont été effectuées dans une gamme de fréquences allant de $10^{-2}$ à $10 \mathrm{~Hz}$. Avant d'effectuer les mesures (rhéologiques, granulométriques et zétamétriques), chaque suspension est agitée magnétiquement pendant une heure. Pour obtenir une meilleure homogénéisation des mélanges sous la géométrie de mesure, tous les fluides étudiés ont été systématiquement soumis aux mêmes conditions avant le test : après la mise en place de l'échantillon (solution de polymère ou mélanges bentonitePEO), on applique un précisaillement, de $10 \mathrm{~s}^{-}$ ${ }^{1}$ durant 2 minutes, suivi par un repos de 2 minutes.

Par ailleurs, une étude bibliographique a montré ( Benchabane 2006) que le pH d'une suspension argileuse est un paramètre déterminant de la charge électrique sur les surfaces des particules. Donc, un changement de $\mathrm{pH}$ modifiera la structure de la suspension et par conséquent, son comportement macroscopique ( Benchabane 2006 ; Ebagninin et al., 2006a ). De ce fait, le pH des suspensions a été contrôlé systématiquement (entre 9,3 et 10,5).

\section{RESULTATS}

\section{Caractérisation rhéologique du poly éthylène oxyde}

Les Figures 1(a, b, c et d) présentent les rhéogrammes des solutions de PEO à différentes concentrations pour les quatre (4) masses moléculaires étudiées.

Ces figures montrent différents comportements rhéologiques dépendant de la concentration et de la masse moléculaire du PEO. On constate que les courbes d'écoulement obtenues ne présentent pas de contrainte seuil. La viscosité des solutions augmente avec la concentration en PEO et la masse moléculaire.

Les résultats de ces essais dynamiques sont représentés sur les Figures $2 \mathrm{a}$ et $2 \mathrm{~b}$.

Il s'agit des variations des modules $\mathrm{G}^{\prime}$ et $\mathrm{G}^{\prime \prime}$ en fonction de la fréquence pour deux concentrations $(2 \%$ et $4 \%$ ) et pour les quatre masses moléculaires de PEO étudiées. Nous remarquons que les deux modules ( $G^{\prime}$ et $\left.G^{\prime \prime}\right)$ augmentent avec la fréquence et la concentration pour toutes les masses moléculaires étudiées. 
En outre, le module visqueux est supérieur au module élastique pour les masses moléculaires $\left(10^{5}\right.$ et $4 \cdot 10^{5}$ g.mol $\left.{ }^{-1}\right)$. Pour ces masses moléculaires, les solutions présentent des propriétés rhéologiques à forte dominante visqueuse. Les mêmes résultats sont obtenus pour les solutions de masse moléculaire $10^{6}$ g.mol ${ }^{-1}$ à des fréquences inférieures à $10 \mathrm{~Hz}$.

En revanche, des croisements de ces deux modules sont observés à des fréquences de $0,03 \mathrm{~Hz}$ et de $0,2 \mathrm{~Hz}$ pour le $\mathrm{PEO}$ de masse moléculaire $4.10^{6}$ g.mol ${ }^{-1}$ et respectivement à des concentrations de 4 et $2 \%$. Les mêmes remarques sont faites pour le PEO de masse moléculaire $10^{6}$ g.mol ${ }^{-1}$, pour la concentration de $4 \%$ et à une fréquence de $10 \mathrm{~Hz}$.

En résumé, on observe que ces graphes présentent des points de croisement $\left(G^{\prime}=G^{\prime \prime}\right)$ : le PEO est plus visqueux qu'élastique à «faible » fréquence (à une fréquence inférieure à la fréquence de croisement) et plus élastique que visqueux à fréquence «élevée » (à une fréquence supérieure à la fréquence de croisement).

Pour mieux comprendre ce phénomène, nous avons étendu l'étude à plusieurs concentrations et à trois fréquences $(0.01,0.1$, et $1 \mathrm{~Hz}$ ) (Figure 3).

Les résultats des modules élastiques (ou de stockage) $\left(G^{\prime}\right)$ et des modules visqueux (ou de perte) $\left(G^{\prime \prime}\right)$ sont exprimés en fonction des concentrations en PEO sur la Figure 3 pour différentes masses moléculaires.

Il est intéressant d'observer sur les différentes courbes que $\mathrm{G}^{\prime \prime}$ augmente avec la masse moléculaire du PEO et la fréquence. On observe que pour les concentrations de PEO de faibles masses moléculaires $\left(10^{5}\right.$ et $4.10^{5}$ g.mol $\left.{ }^{-1}\right)$, les valeurs de $\left(G^{\prime \prime}\right)$ sont supérieures à celles de $\left(G^{\prime}\right)$ sur les gammes de fréquences et de concentrations étudiées. Pour des masses moléculaires élevées $\left(10^{6}\right.$ et $\left.4.10^{6} \mathrm{~g} \cdot \mathrm{mol}^{-1}\right)$, les valeurs de $\left(G^{\prime \prime}\right)$ sont supérieures à celles de $\left(G^{\prime}\right)$ à des faibles fréquences. Des croisements de ces deux paramètres sont observés à des concentrations données et les valeurs de $\left(G^{\prime}\right)$ sont supérieures à celles de $\left(G^{\prime \prime}\right)$ à des fréquences importantes et à des concentrations élevées.

Influence du PEO sur les suspensions d'argile (mélange argile-polymère)

Les Figures 4 ( $a, b$, c et d) présentent les courbes d'écoulement de la suspension de bentonite de base (suspension de bentonite à $5 \%$ ) et des mélanges bentonite-PEO à différentes concentrations et à différentes masses moléculaires $\left(10^{5}, 4.10^{5}, 10^{6}\right.$ et $4.10^{6}$ g.mol $\left.{ }^{-1}\right)$.

On observe que pour les masses moléculaires de $4.10^{5}, 10^{6}$ et $4.10^{6} \mathrm{~g} \cdot \mathrm{mol}^{-1}$, l'ajout de PEO, même à faibles concentrations, change considérablement le comportement rhéologique de la suspension de base (Figures 4b, 4c et 4d).

En revanche, l'ajout de PEO à $10^{5}$ g.mol ${ }^{-1}$ n'entraîne pas d'augmentation de viscosité sur la majeure partie de la gamme de vitesses de cisaillement $\left(\dot{\gamma}>5 s^{-1}\right)$ (Figure 4a). Par contre, nous remarquons une augmentation de la viscosité pour une concentration de PEO de $0,1 \%$ suivie d'une diminution pour les autres concentrations (Figure 4a).

En outre, On note l'existence d'une contrainte seuil pour tous les mélanges "bentonite-PEO", ce qui montre que la nature structurale de la suspension de base est modifiée après l'ajout de polymère (Figures 4) (Ebagninin, 2006, 2007a,b, 2008). De même, la viscosité des mélanges augmente avec la concentration et la masse moléculaire des polymères $\left(4.10^{5}, 10^{6}\right.$ et $\left.4.10^{6} \mathrm{~g} \cdot \mathrm{mol}^{-1}\right)$. Cette augmentation de la viscosité pourrait s'expliquer par le fait que l'ajout du poly éthylène oxyde entraîne la formation d'une structure plus ou moins rigide pour la masse moléculaire de $4 \cdot 10^{5} \mathrm{~g} \cdot \mathrm{mol}^{-1}$. Cette structure devient de plus en plus rigide avec la longueur des chaînes polymériques pour les masses 
moléculaires les plus élevées $\left(10^{6}\right.$ et $4.10^{6}$ g.mol ${ }^{-1}$ ).

Pour mieux comprendre la structure responsable des comportements rhéologiques et caractériser l'état des interactions bentonitePEO, il est indispensable d'effectuer des mesures non destructives à faibles contraintes de cisaillement. Pour cela, des essais viscoélastiques ont été effectués sur la bentonite de base et les mélanges bentonitePEO étudiés.

Les essais dynamiques ont été réalisés pour mieux comprendre le mécanisme responsable de ces comportements rhéologiques (écoulement). Ces mesures permettent de caractériser l'état des interactions entre les chaînes de polymère et les particules argileuses en accédant aux propriétés des suspensions dans des conditions proches de l'état de repos, sans en détruire la structure interne.

La Figure 5 présente les variations du module élastique $G^{\prime}$ et le module visqueux $G^{\prime \prime}$ en fonction de la fréquence pour la suspension de base (bentonite 5\%) et les mélanges bentonite de base-PEO à une concentration de $0,05 \%$ et pour les quatre masses moléculaires. Plusieurs observations peuvent être faites :

a) pour toutes les suspensions, le module élastique $\mathrm{G}^{\prime}$ est supérieur au module visqueux G", ce qui indique une prépondérance du caractère élastique sur le caractère visqueux dans les mélanges ;

b) pour les masses moléculaires de $4.10^{5}$ et $4.10^{6}$ g.mol ${ }^{-1}$, les deux modules $\mathrm{G}^{\prime}$ et G" augmentent lorsque l'on ajoute le polymère à la suspension de base ;

c) pour le PEO de plus faible masse moléculaire $\left(10^{5} \mathrm{~g} \cdot \mathrm{mol}^{-1}\right)$, le module visqueux de la suspension de base est confondu avec celui du mélange. En revanche, le module élastique de la suspension de base est supérieur à celui du mélange. Cela est le signe qu'à cette masse moléculaire et à cette concentration $(0,05 \%)$, le polymère modifie la microstructure de la suspension argileuse ; d) pour les quatre masses moléculaires, le module élastique G' est indépendant de la fréquence, ce qui est significatif d'un comportement de type gel: le polymère de masse moléculaire élevée provoque de fortes interactions entre les particules argileuses.

Pour mieux voir les variations des deux modules ( $G$ ' et $G$ ) sur toutes les gammes de concentrations et de masses moléculaires en PEO étudiées, ces mêmes courbes sont représentées en fixant les fréquences à 0.01 , 0.1 et $1 \mathrm{~Hz}$ pour les différentes masses moléculaires et en fonction des concentrations en PEO.

Les Figures $6(\mathrm{a}, \mathrm{b}$ et $\mathrm{c})$ présentent la variation des modules $G^{\prime}$ et $G^{\prime \prime}$ des mélanges bentonite- PEO de différentes masses moléculaires en fonction de la concentration. De manière générale, le module élastique G' est supérieur au module visqueux $G^{\prime \prime}$. L'écart entre ces deux paramètres devient plus important lorsque la fréquence augmente. On observe que, pour les mélanges bentonitePEO $\left(4.10^{5}, 10^{6}\right.$ et $4.10^{6}$ g.mol $\left.{ }^{-1}\right), G^{\prime}$ et $\mathrm{G}^{\prime \prime}$ augmentent avec la concentration de polymère pour les trois (3) fréquences représentées. L'allure des courbes est quasi-identique, notamment pour les concentrations de polymère supérieures à une valeur d'environ $0,02 \%$. Pour ces concentrations, $G^{\prime}$ et $G^{\prime \prime}$ ne varient pas avec la fréquence.

Pour les mélanges bentonite-PEO de plus faible masse moléculaire $\left(10^{5} \mathrm{~g} \cdot \mathrm{mol}^{-1}\right)$, on observe un module visqueux $G^{\prime \prime}$ qui ne varie pas avec la concentration en polymère. On observe également une diminution du module élastique G' avec l'augmentation de la concentration en polymère (pour la fréquence la plus faible) qui reste ensuite pratiquement constant (pour des fréquences importantes). Ceci confirme les résultats établis en écoulement où l'on observe une augmentation de la viscosité pour une concentration de $0,1 \%$ suivi d'une diminution. 
Tableau 1: Constante du modèle de Cross pour les solutions de masse moléculaire : a) $4.10^{5}$ g.mol ${ }^{-1}$ et b) $10^{6} \mathrm{~g} \cdot \mathrm{mol}^{-1}$.

a) PEO $4 \cdot 10^{5}$ g.mol ${ }^{-1}$

\begin{tabular}{|c|c|c|c|c|}
\hline $\begin{array}{l}\text { Conc. } \\
{[\%]}\end{array}$ & $\begin{array}{l}\lambda \\
{[\mathrm{s}]}\end{array}$ & $\begin{array}{l}m \\
{[-]}\end{array}$ & $\begin{array}{l}\eta_{0} \\
{[\text { Pa.s] }}\end{array}$ & $\begin{array}{l}\eta_{\infty} \\
{[\text { Pa.s] }}\end{array}$ \\
\hline 0.25 & 0.000886 & 0.698521 & 0.00227 & 0.0016 \\
\hline 0.5 & 0.000585 & 0.788291 & 0.0045 & 0.0025 \\
\hline 1 & 0.001161 & 0.786083 & 0.0123 & 0.0042 \\
\hline 2 & 0.003255 & 0.731944 & 0.049 & 0.008 \\
\hline 3 & 0.012745 & 0.694578 & 0.255 & 0.011 \\
\hline 4 & 0.025663 & 0.673020 & 0.65 & 0.018 \\
\hline 6 & 0.074003 & 0.65446 & 4.2 & 0.031 \\
\hline 8 & 0.176655 & 0.634374 & 17 & 0.035 \\
\hline 10 & 0.293663 & 0.634896 & 55 & 0.045 \\
\hline
\end{tabular}

b) PEO $10^{6} \mathrm{~g} \cdot \mathrm{mol}^{-1}$

\begin{tabular}{lllll}
\hline $\begin{array}{l}\text { Conc. } \\
{[\%]}\end{array}$ & $\begin{array}{l}\lambda \\
{[\mathrm{s}]}\end{array}$ & $\begin{array}{l}\mathrm{m} \\
{[-]}\end{array}$ & $\begin{array}{l}\eta_{0} \\
{[\text { Pa.s }]}\end{array}$ & $\begin{array}{l}\eta_{\infty} \\
{[\text { Pa.s }]}\end{array}$ \\
\hline 0.5 & 0.00112 & 0.89 & 0.01009 & 0.0032 \\
1 & 0.0048 & 0.78 & 0.0419 & 0.006 \\
2 & 0.0501 & 0.64 & 0.33 & 0.012 \\
3 & 0.0981 & 0.67 & 1.74 & 0.02 \\
4 & 0.1998 & 0.68 & 7.1 & 0.03 \\
6 & 0.61392 & 0.686 & 70 & 0.045 \\
8 & 1.30247 & 0.67 & 275 & 0.055 \\
10 & 3.445 & 0.62 & 1300 & 0.06 \\
\hline
\end{tabular}


Tableau 2: Les constantes du modèle de Cross pour la solution de PEO de masse moléculaire $4.10^{6}$ g. $\mathrm{mol}^{-1}$.

\begin{tabular}{lllll}
\hline $\begin{array}{l}\text { Conc. } \\
{[\%]}\end{array}$ & $\begin{array}{l}\lambda \\
{[\mathrm{s}]}\end{array}$ & $\mathrm{m}$ & $\begin{array}{l}\eta_{0} \\
{[-]}\end{array}$ & $\begin{array}{l}\eta_{\infty} \\
{[\mathrm{Pa} . \mathrm{s}]}\end{array}$ \\
\hline 0.1 & 0.00575552 & 0.926939955 & 0.0035 & 0.0022 \\
0.25 & 0.01611851 & 0.77186654 & 0.0131 & 0.004 \\
0.5 & 0.08359314 & 0.746191179 & 0.097 & 0.0089 \\
0.8 & 0.26649181 & 0.68531908 & 0.51 & 0.013 \\
1 & 0.47180181 & 0.715295629 & 1.5 & 0.02 \\
1.5 & 1.41998647 & 0.741999547 & 11 & 0.035 \\
2 & 2.6644691 & 0.756473711 & 45 & 0.053 \\
2.5 & 3.49864511 & 0.779496591 & 122 & 0.065 \\
3 & 4.73146336 & 0.780959351 & 240 & 0.095 \\
4 & 7.79893368 & 0.801254214 & 695 & 0.1 \\
5 & 15.391133 & 0.758959252 & 3104.04552 & 0.15 \\
\hline
\end{tabular}

a) Masse moléculaire $: 10^{5} \mathrm{~g} \mathrm{~mol}^{-1}$

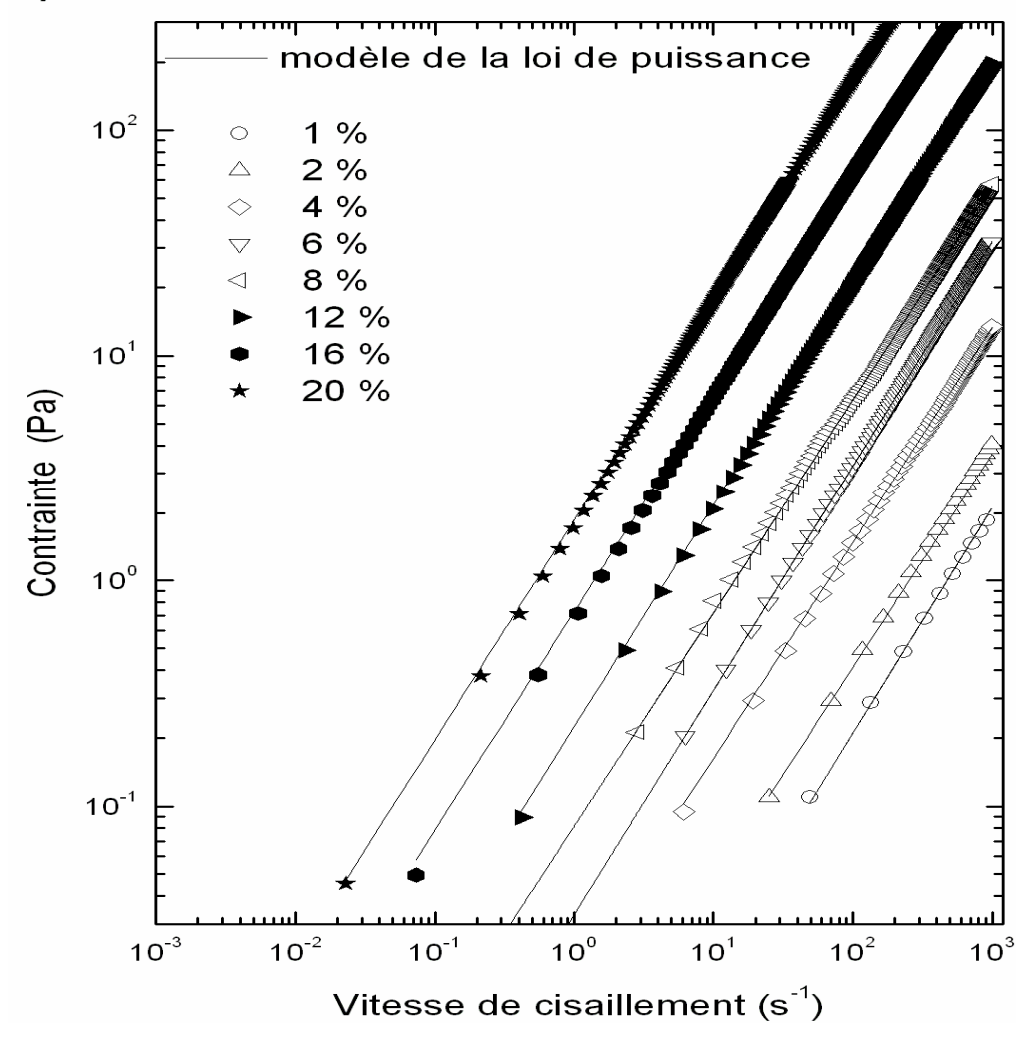


b) Masse moléculaire : $4.10^{5} \mathrm{~g} \mathrm{~mol}^{-1}$

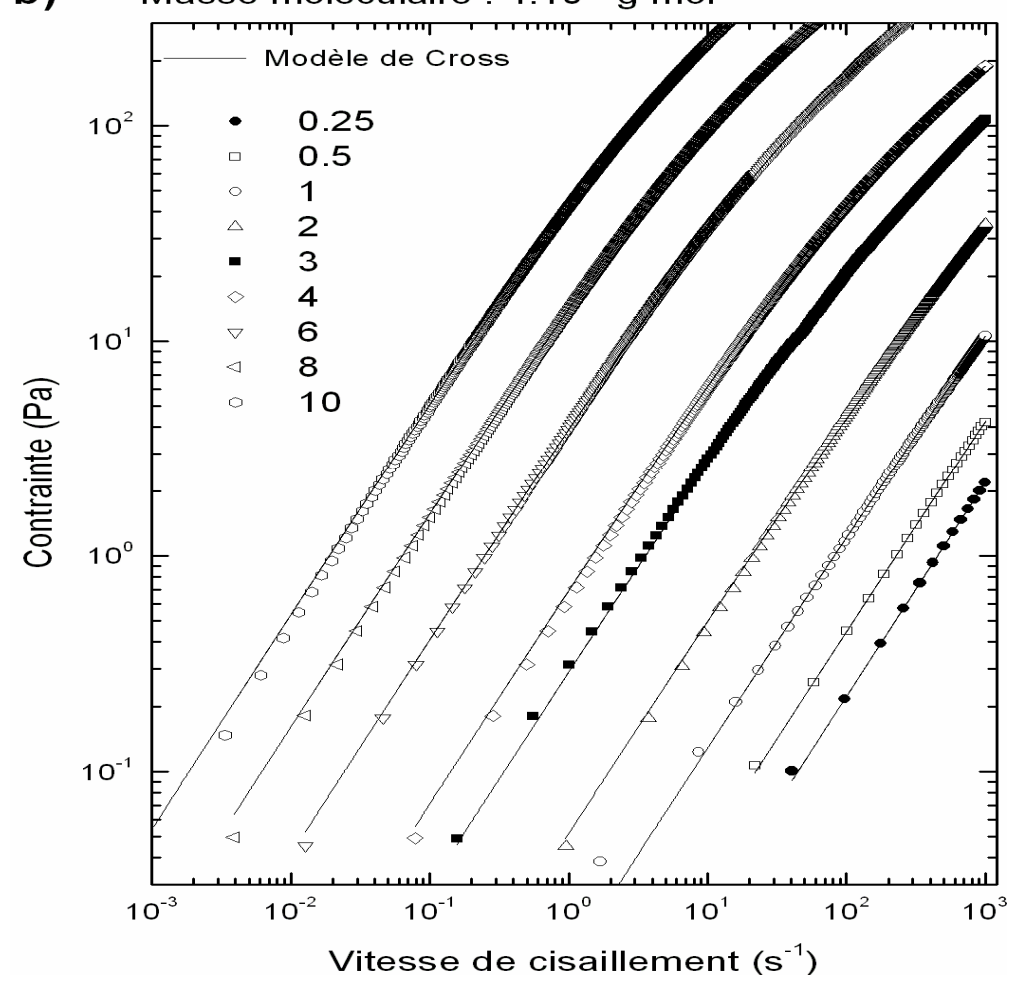

c) Masse moléculaire : $10^{6} \mathrm{~g} \mathrm{~mol}^{-1}$

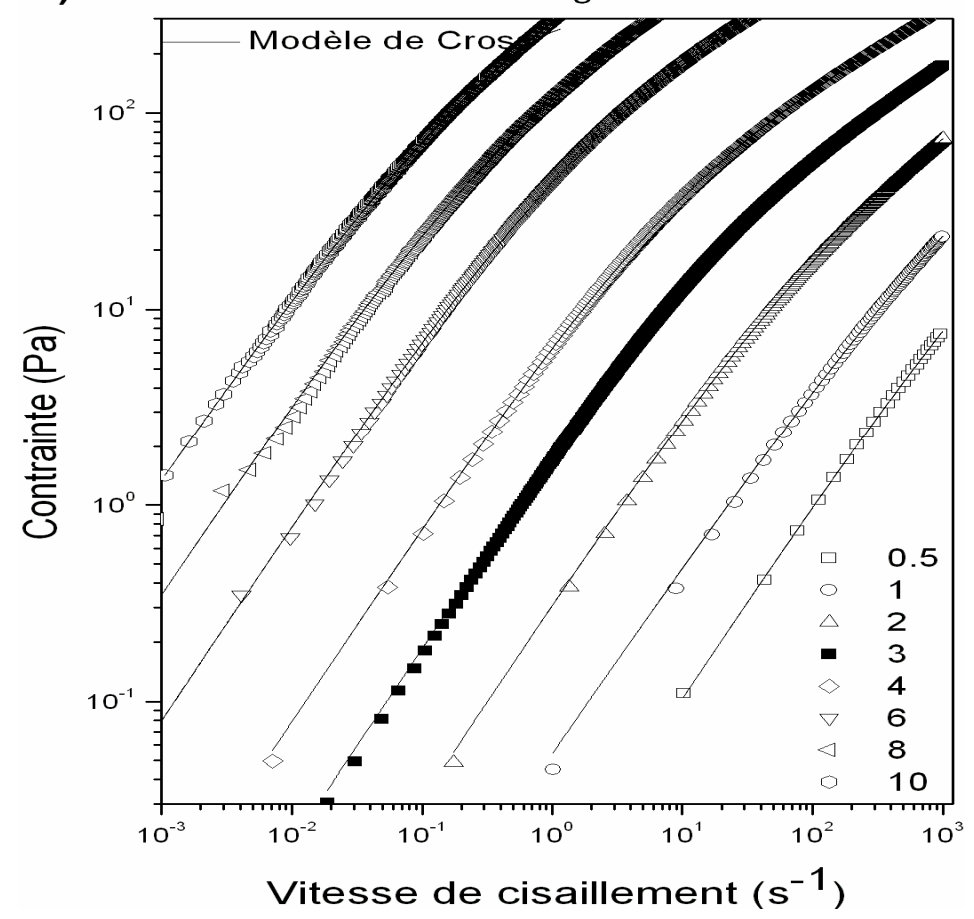


d) Masse moléculaire : $410^{6} \mathrm{~g} \mathrm{~mol}^{-1}$ Modèle de Cross

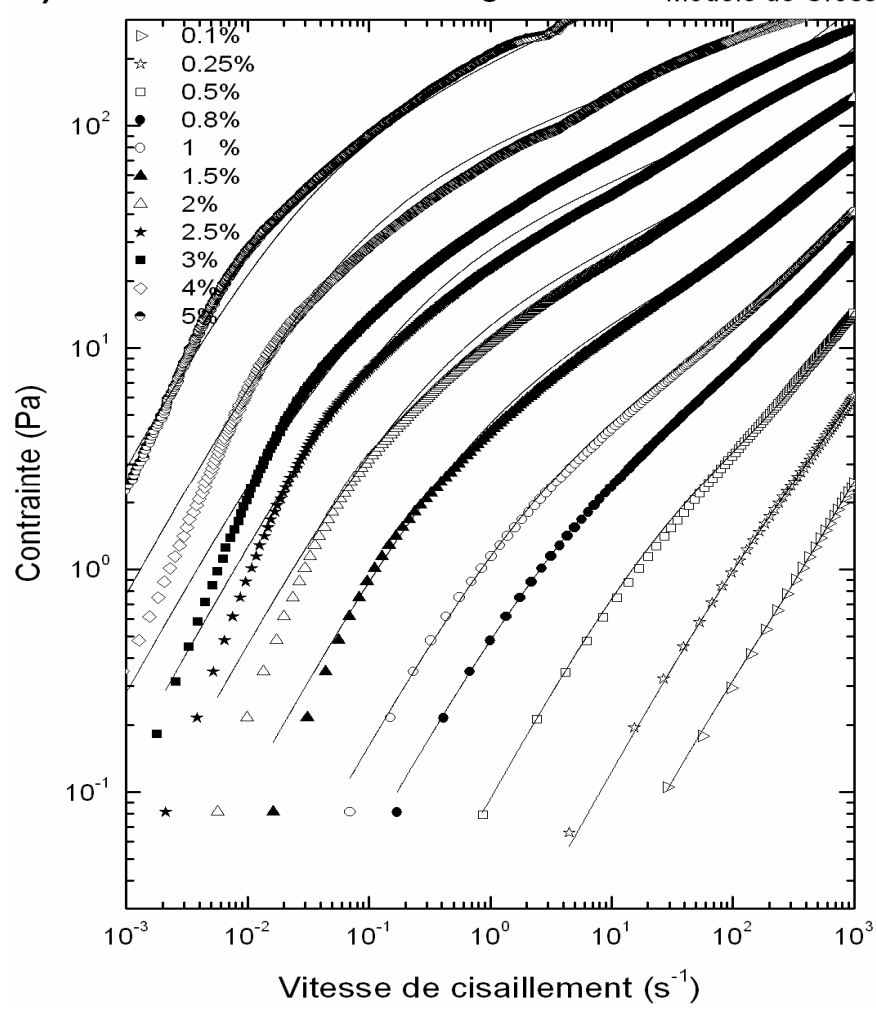

Figure 1 : Courbes d'écoulement des solutions de PEO de différentes masses moléculaires $10^{5}$, $4.10^{5}, 10^{6}$ et $4.10^{6}$ g.mol ${ }^{-1}$ à différentes concentrations.

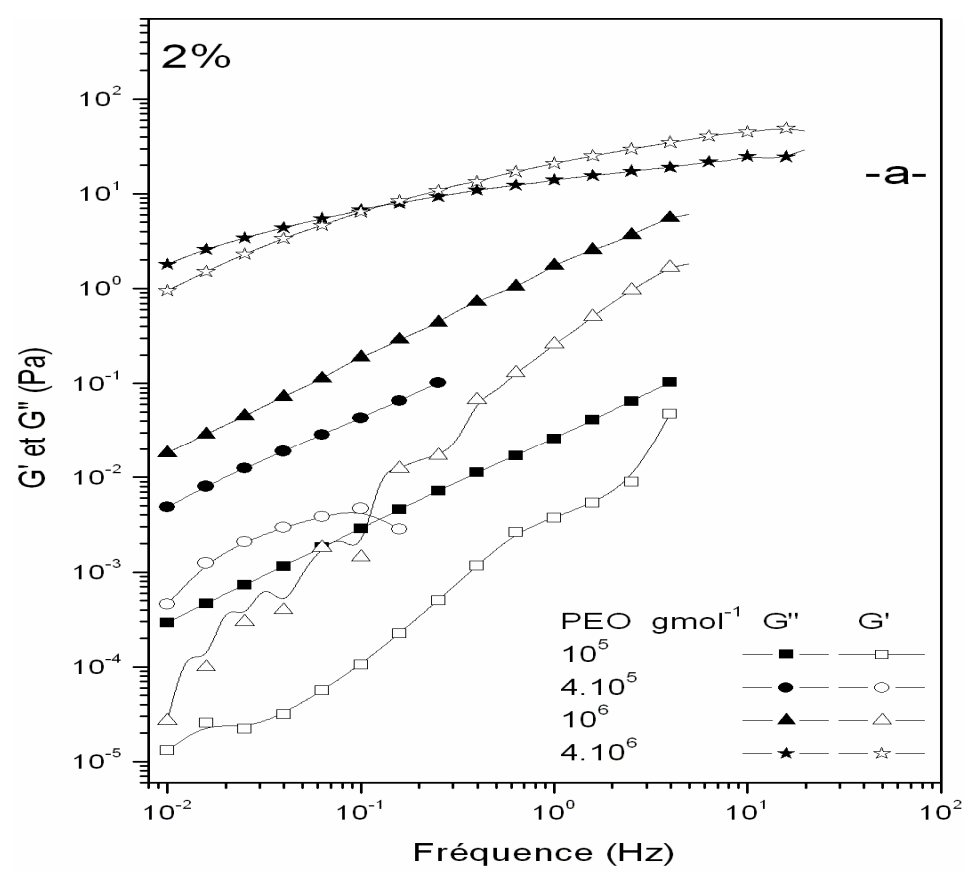




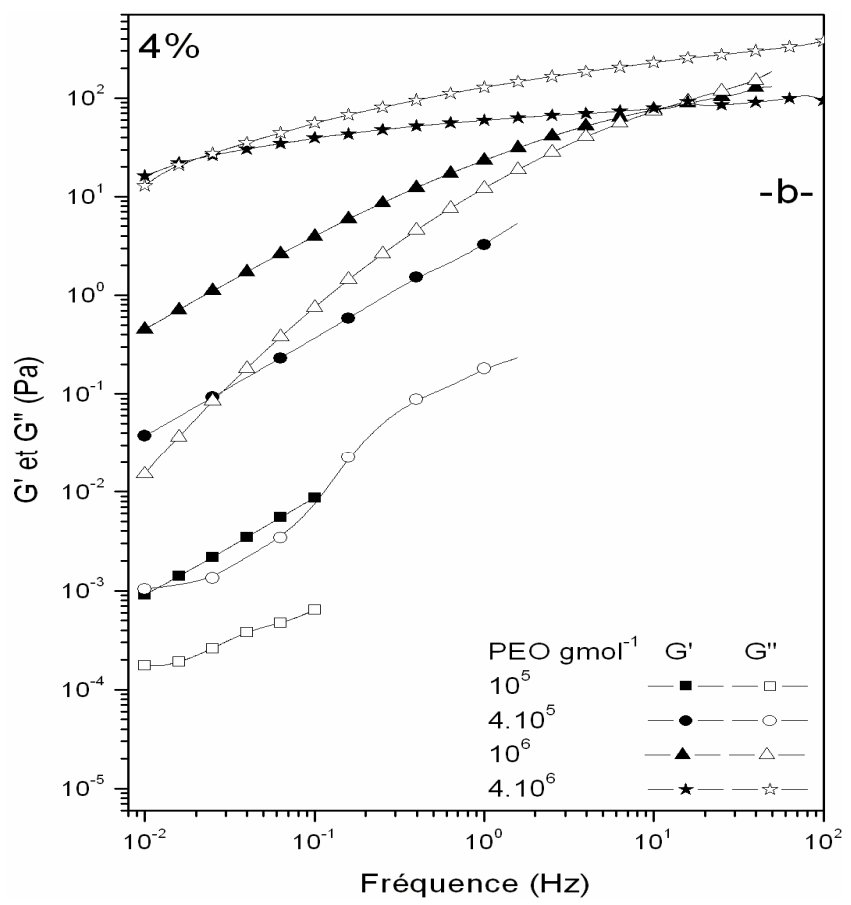

Figure 2 : Variation des modules de perte $G^{\prime \prime}$ et de conservation $G^{\prime}$ en fonction de la fréquence pour les concentrations 2 et $4 \%$ et pour les différentes masses moléculaires.

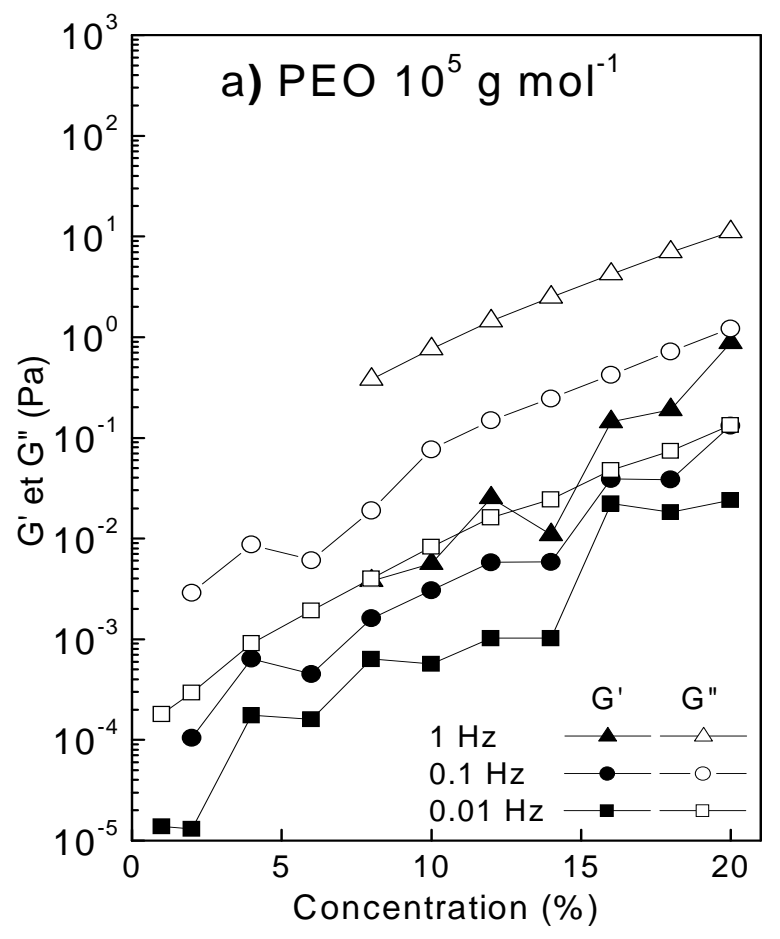



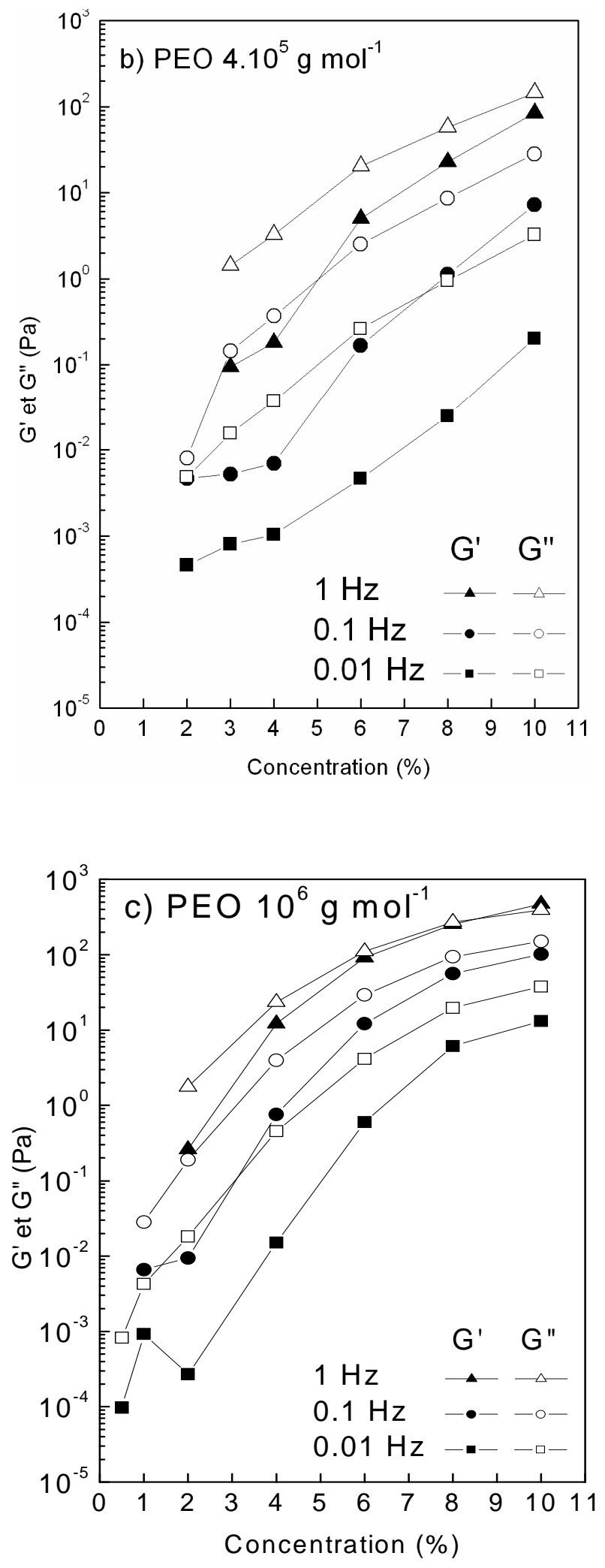


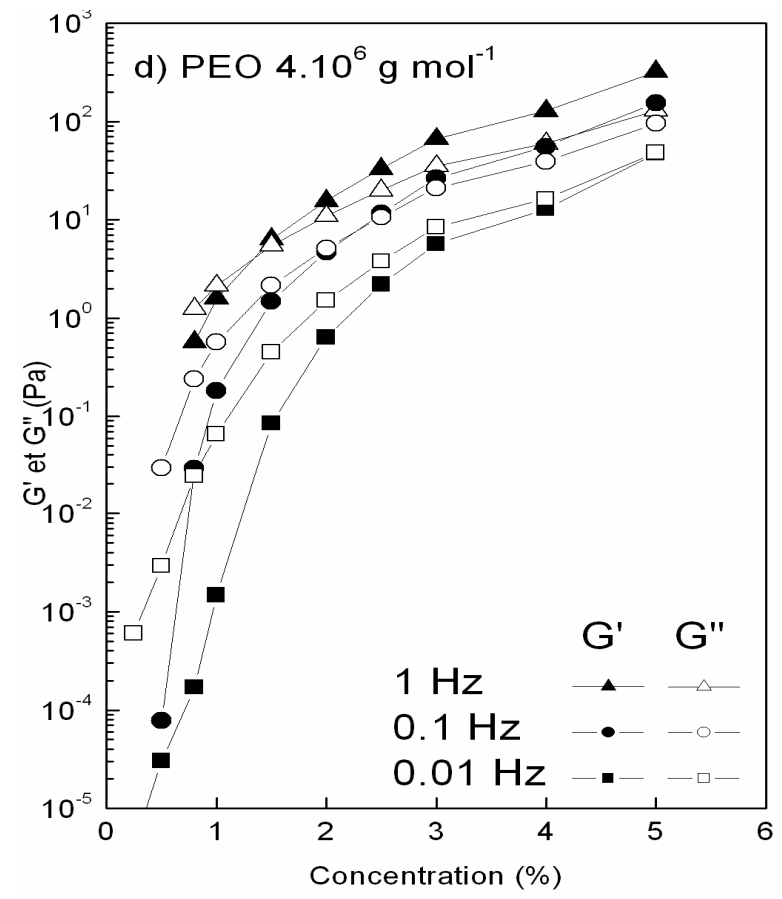

Figure 3 : Variation des modules de perte et de conservation en fonction de la concentration en PEO à différentes fréquences et à différentes masses moléculaires.

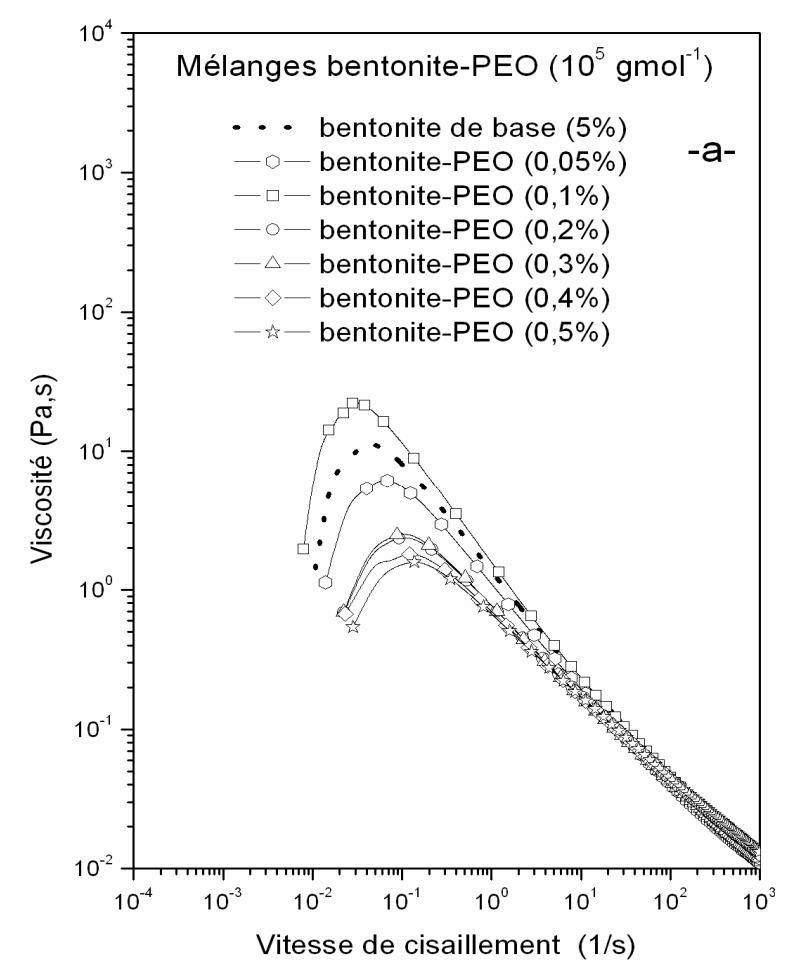



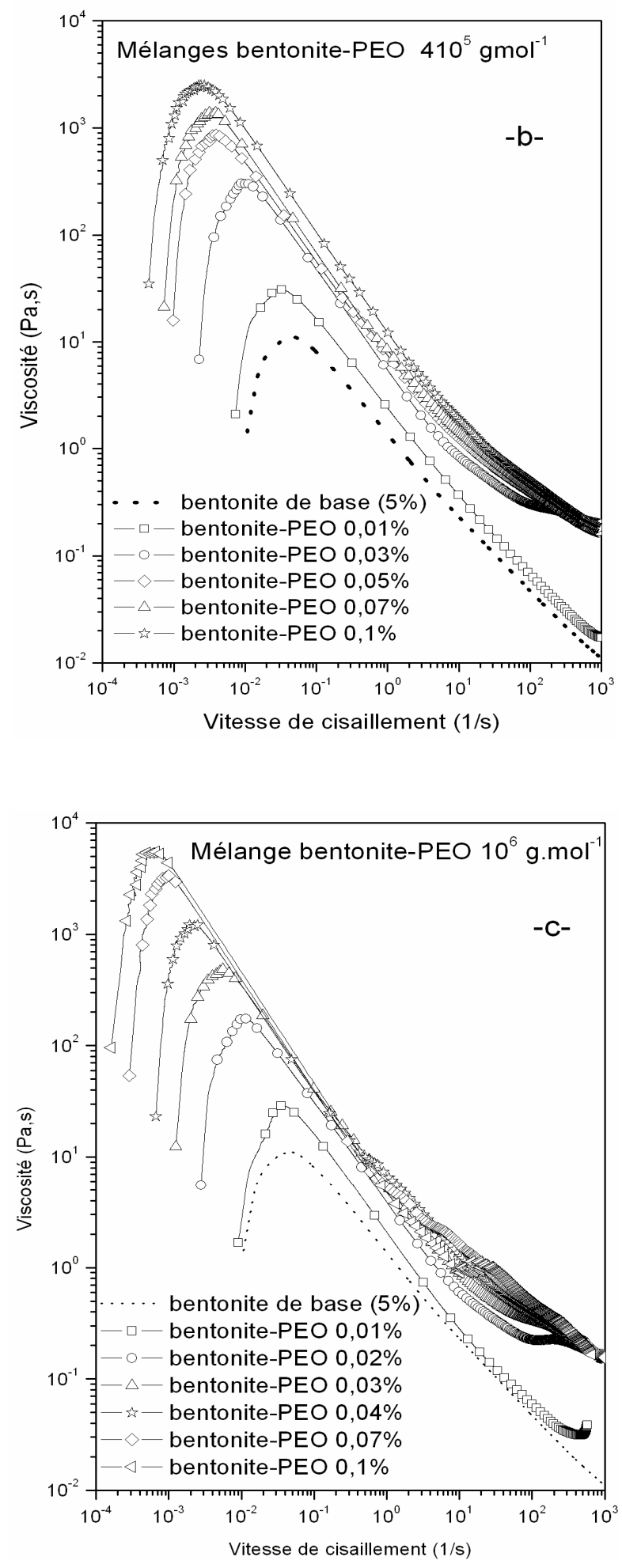


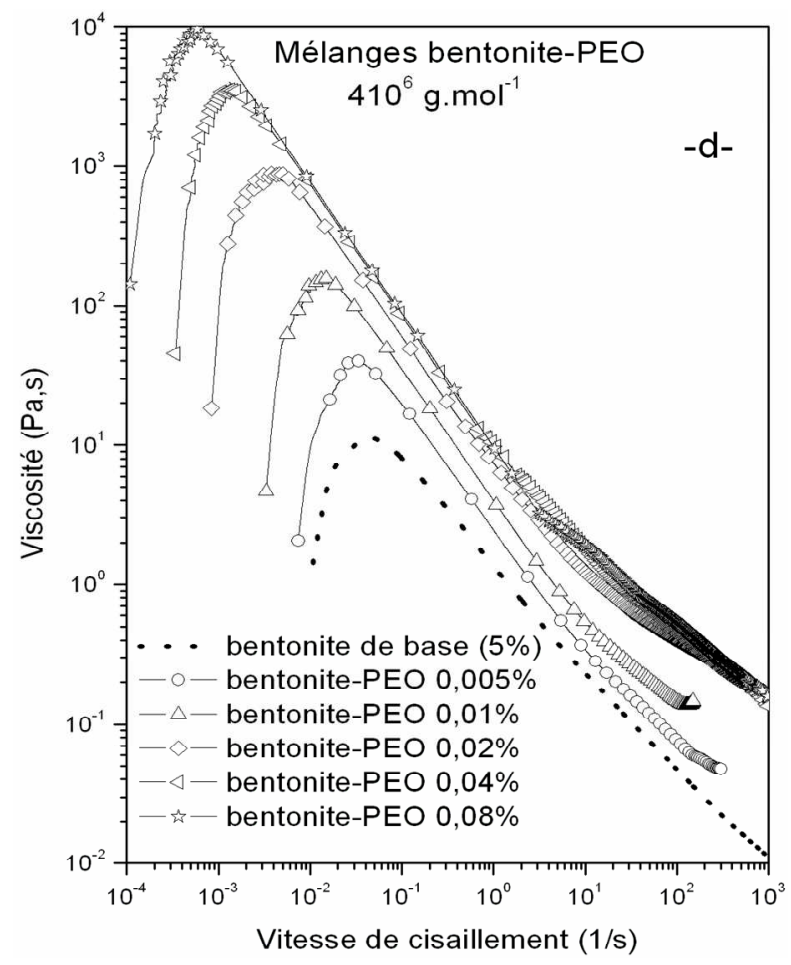

Figure 4 : Rhéogrammes de la suspension de base et des mélanges bentonite-PEO de différentes masses moléculaires $\left(10^{5}, 4 \cdot 10^{5}, 10^{6}\right.$ et $\left.4 \cdot 10^{6} \mathrm{~g} \cdot \mathrm{mol}^{-1}\right)$ à différentes concentrations.

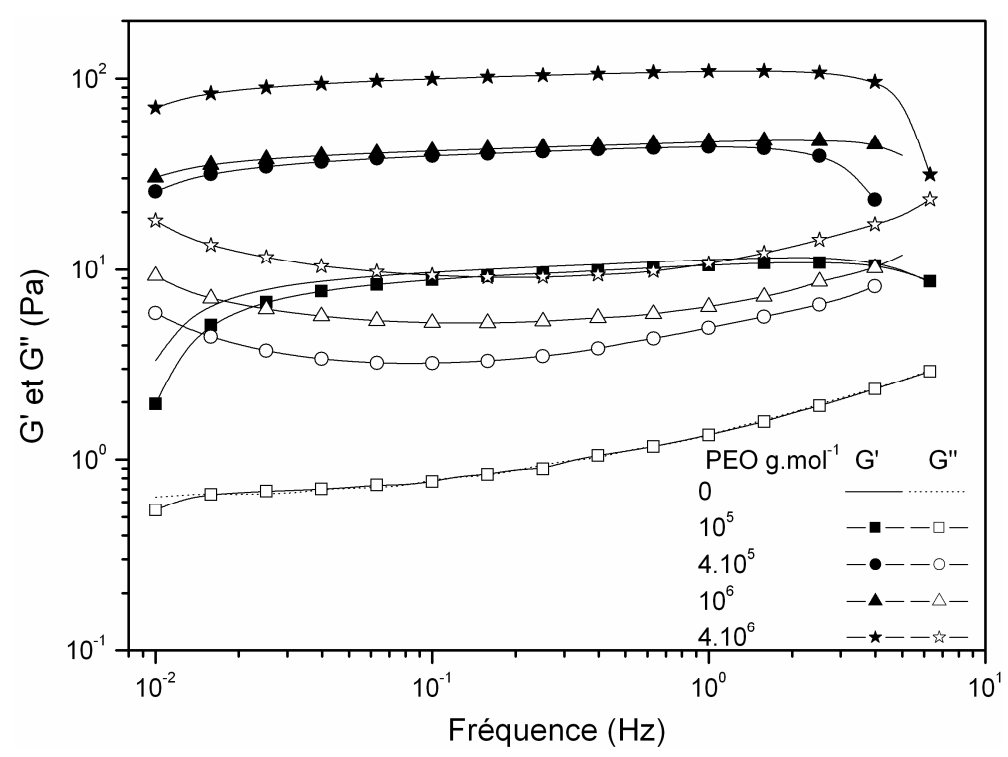

Figure 5 : Courbes d'oscillation de $\mathrm{G}^{\prime}$ et $\mathrm{G}^{\prime \prime}$ en fonction de la fréquence pour la bentonite à $5 \%$ et du mélange bentonite $5 \%+$ PEO à $0,05 \%$ pour les 4 masses moléculaires $\left(10^{5}, 410^{5}, 10^{6}\right.$ et $4.10^{6}$ g.mol $\left.{ }^{-1}\right)$. 

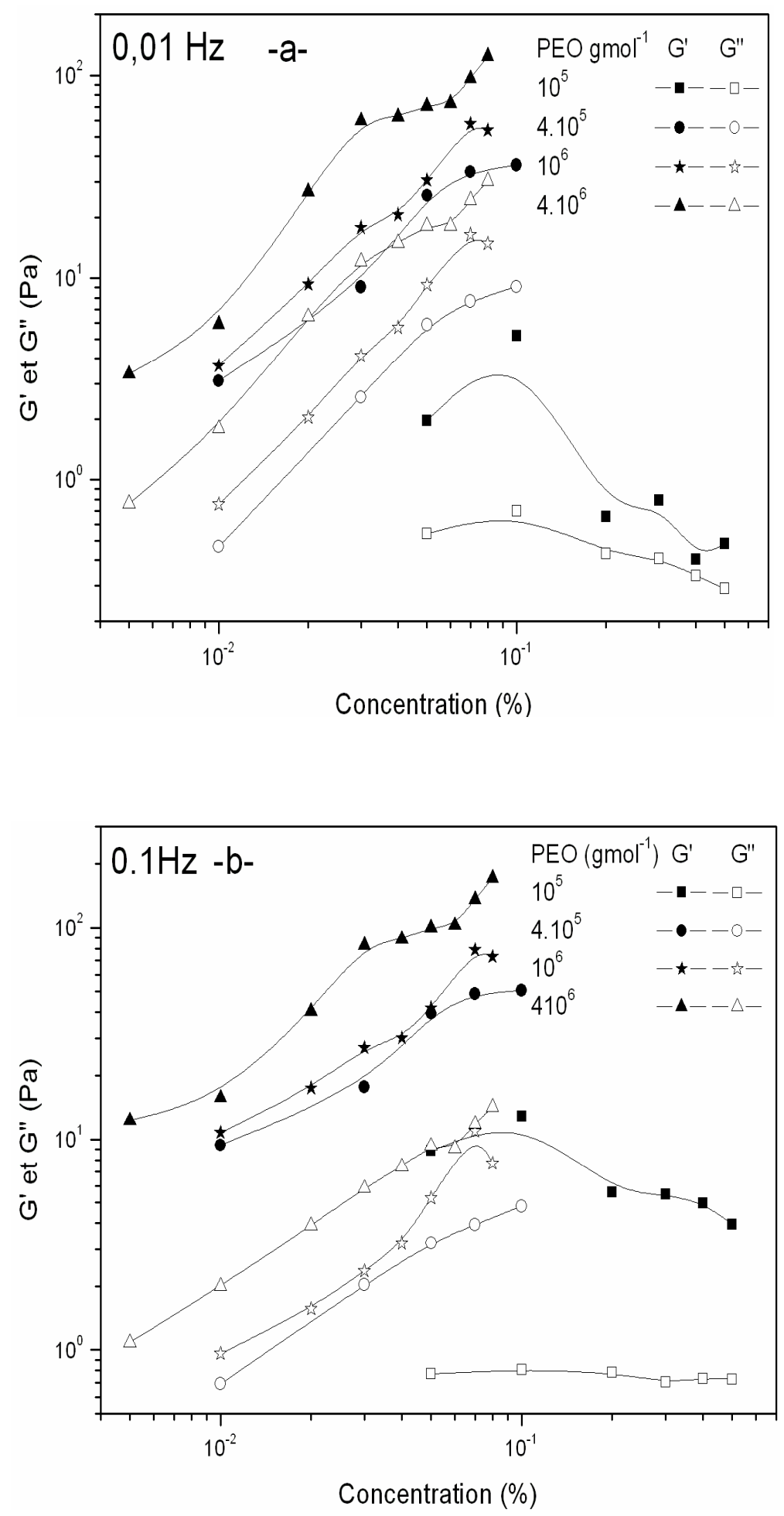


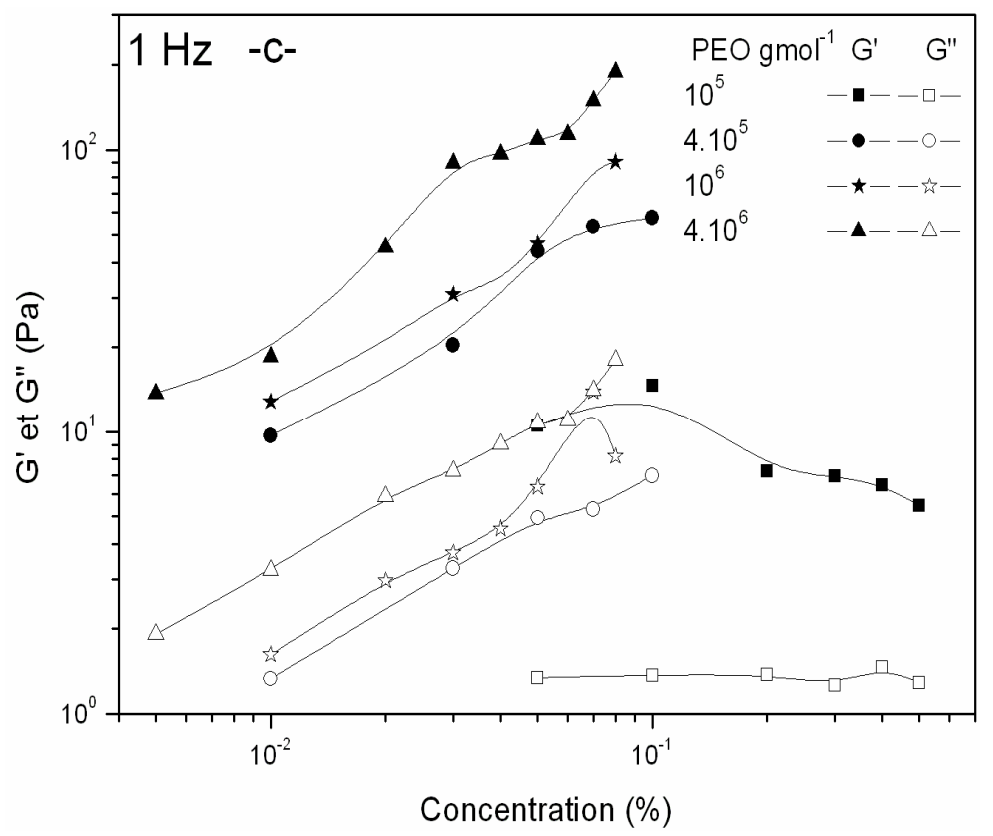

Figure 6 : Modules de conservation $\mathrm{G}^{\prime}$ et de perte $\mathrm{G}^{\prime}$ des mélanges bentonite-PEO en fonction de la concentration en PEO, pour différents masses moléculaires.

\section{DISCUSSION}

Ces résultats (rhéogrammes des solutions de polyéthylène oxyde) concordent avec ceux obtenus antérieurement par d'autres chercheurs (Briscoe et al., 1998; Gauri et Koelling, 1997 ; Kalashnikov, 1994 ; Kang et al., 2005 ; Ortiz et al., 1994 ; Powell et Schwarz, 1975 ; Powell, 1979).

On interprète généralement ces résultats (solutions de polyéthylène oxyde) par le fait que les propriétés rhéologiques des solutions de polymères sont significativement affectées par les interactions polymère-solvant dans les solutions diluées et polymèrespolymères dans les solutions concentrées.

Généralement, la théorie présentée pour expliquer l'augmentation de la viscosité en fonction de la concentration et de la masse moléculaire du polymère se résume de la manière suivante: pour des solutions de polymère relativement concentrées, l'augmentation de la vitesse de cisaillement modifie l'ordre des molécules dans le milieu. Les chaînes de polymères sont initialement très enchevêtrées, ce qui confère à la solution une grande viscosité au repos (ou aux faibles vitesses de cisaillement). L'augmentation de cette vitesse de cisaillement permet aux chaînes moléculaires de s'étirer et de s'orienter en formant des couches parallèles dans la solution. Cet alignement moléculaire facilite le glissement entre les chaînes de polymères et entraîne la réduction de la viscosité (rhéofluidification). Ce degré d'enchevêtrement diminue avec la masse moléculaire du polymère (Benchabane, 2006; Briscoe et al., 1998 ,1996 ; Ortiz et al., 1997 ; Rossi et al., 1997).

En outre, on observe que la forme des courbes d'écoulement change lorsque la masse moléculaire augmente et tout particulièrement pour la masse moléculaire la plus élevée $\left(4.10^{6}\right.$ g.mol $\left.{ }^{-1}\right)$. Pour la masse moléculaire la plus faible $\left(10^{5} \mathrm{~g} \cdot \mathrm{mol}^{-1}\right)$, les rhéogrammes sont linéaires (ou quasiment) (Figure 1a). La viscosité ne dépend pas (ou très faiblement) de la vitesse de cisaillement. La Figure 1a montre le comportement des solutions de PEO de faible masse moléculaire $\left(10^{5} \mathrm{~g} \cdot \mathrm{mol}^{-1}\right)$. Celui-ci peut être décrit par le 
modèle de la loi de puissance, proposé par Ostwald-de Waele (Benchabane, 2006) : $\tau=k \cdot \dot{\gamma}^{n}$, où $\tau[\mathrm{Pa}], k\left[\mathrm{~Pa}_{\mathrm{s}}{ }^{n}\right]$ et $n$ sont respectivement la contrainte de cisaillement, la consistance et l'indice d'écoulement, $\dot{\gamma}$ est la vitesse de cisaillement. A cette masse moléculaire $\left(10^{5}\right.$ g.mol $\left.{ }^{-1}\right)$ donc, le comportement rhéologique de la solution de PEO est quasi-Newtonien puisque l'indice d'écoulement $n$ reste très proche de l'unité pour toutes les concentrations étudiées $(0,94<$ $n<1)$.

Pour les masses moléculaires $4.10^{5}$ et $10^{6}$ g.mol ${ }^{-1}$, les données corrèlent parfaitement avec le modèle de Cross :

$\frac{\eta-\eta_{\infty}}{\eta_{0}-\eta_{\infty}}=\frac{1}{1+(\lambda \cdot \dot{\gamma})^{m}}$ Où $\lambda$ est un temps caractéristique de relaxation, $\eta_{0}$

et $\eta_{\infty}$ sont respectivement les viscosités à cisaillements nul et infini en [Pa.s] et $m$ représente le degré d'agrégation et de désagrégation de la suspension.

Pour toutes les concentrations étudiées et sur toute la plage de vitesses de cisaillement (Figures $1 \mathrm{~b}$ et 1c, respectivement), les paramètres du modèle de Cross sont résumés dans les Tableaux 1 et 2 . Le temps caractéristique $\lambda$, les viscosités à cisaillement nul $\eta_{0}$ et infini $\eta_{\infty}$ augmentent avec la concentration en PEO. En revanche, les valeurs de l'indice $m$ restent pratiquement constantes.

L'augmentation de la viscosité à cisaillements nul et infini est très importante pour les concentrations élevées de polymères et cela, en raison des fortes interactions entre les chaînes polymériques.

L'augmentation du temps caractéristique $\lambda$ (considéré comme un temps de relaxation), avec les concentrations en PEO montre que l'équilibre entre les processus d'enchevêtrement et de désenchevêtrement (liés à la vitesse de cisaillement) prend plus de temps (lorsque les concentrations en polymère augmentent).
Des résultats viscoélastiques identiques ont été obtenus par plusieurs auteurs (Clasen et Kulicke, 2001 ; Habas et al., 2008 ; Moura et al., 2007 ; Niedzwiedz et al., 2008 ; Coussot et Gossiord, 2001). On peut comprendre aisément l'origine d'un tel comportement rhéologique en remarquant que les chaînes macromoléculaires adjacentes forment des boucles d'enchevêtrement topologiques les unes avec les autres: pour les «faibles» fréquences. La durée de la période de cisaillement est suffisamment longue pour permettre aux boucles de se dénouer, si bien que le polymère présente des propriétés liquides. A l'inverse, pour les «hautes» fréquences, la période est très courte pour que les enchevêtrements se défassent, si bien que le polyéthylène oxyde a tendance à se comporter comme un réseau réticulé (les boucles d'enchevêtrement jouant le rôle de liaisons chimiques).

Les résultats des modules élastiques (ou de stockage) $\left(G^{\prime}\right)$ et des modules visqueux (ou de perte) $\left(G^{\prime \prime}\right)$ effectuées sur ces solutions de (PEO) sont exprimés en fonction des concentrations en PEO pour différentes masses moléculaires (à des fréquences données) (Figure 3).

Pour les concentrations de PEO de faibles masses moléculaires $\left(10^{5}\right.$ et $4.10^{5}$ g.mol $\left.{ }^{-1}\right)$, les valeurs de $\left(G^{\prime \prime}\right)$ sont supérieures à celles de $\left(G^{\prime}\right)$ sur les gammes de fréquences et de concentrations étudiées. Ce qui signifie que les propriétés visqueuses sont prépondérantes par rapport aux propriétés élastiques. Ces résultats peuvent être prédits à partir des résultats des courbes d'écoulement (Figure 1a et 1b). Les solutions sont essentiellement Newtoniennes sur toutes les concentrations de la masse moléculaire $10^{5}$ g.mol ${ }^{-1}$ et $410^{5}$ g. $\mathrm{mol}^{-1}$ (à des concentrations inférieures ou égales à $2 \%$ ), avec un comportement purement visqueux.

Toutefois, pour des concentrations de PEO supérieures à $2 \%$, le comportement devient non-Newtonien (Figure 1b). Même si $\left(\mathrm{G}^{\prime \prime}\right)$ reste toujours supérieur à $\left(G^{\prime}\right)$ sur toutes 
les gammes de fréquences, l'intervalle entre ces deux valeurs diminue avec l'augmentation de la concentration (les propriétés visqueuses baissent au profit des propriétés élastiques).

Pour toutes les masses moléculaires étudiées, les modules de pertes sont supérieurs aux modules de conservations (à de faibles fréquences). Ceci pourrait s'expliquer par le fait que les déformations du matériau sont très faibles (à des faibles fréquences), l'essentiel de l'énergie est dissipée (à cause du comportement visqueux). Les chaînes de polymère disposent de suffisamment de temps pour éviter la déformation imposée (extérieurement) par la relaxation vers un état énergétiquement favorable. Cette relaxation se met en place par patinage des points d'enchevêtrement des chaînes de polymères entrelacées (Clasen et Kulicke, 2001). Le comportement rhéologique des solutions de PEO à des masses moléculaires importantes $\left(10^{6}\right.$ et $4.10^{6}$ g.mol $\left.{ }^{-1}\right)$ passent du domaine visqueux $\left(\left(\mathrm{G}^{\prime \prime}\right)>\left(G^{\prime}\right)\right)$ à un domaine élastique $\left(\left(\mathrm{G}^{\prime \prime}\right)<\left(G^{\prime}\right)\right)$, d'une part lorsque la concentration augmente à des fréquences données et d'autre part lorsque la fréquence augmente à des concentrations données.

Puisque la fréquence augmente, le temps de relaxation disponible décline. Les chaînes de polymère ne peuvent plus glisser les unes sur les autres de sorte que le point d'enchevêtrement tende de plus en plus vers un point fixe du réseau. La conséquence est que, la capacité de ce réseau provisoire de polymère à stocker de l'énergie imposée augmente.

Contrairement au module de perte, le module de stockage augmente brusquement avec la fréquence, les deux courbes se croisent et les composants élastiques deviennent supérieurs aux composants visqueux.

Le point d'intersection des deux courbes, $G^{\prime}(\omega)=G^{\prime \prime}(\omega)$ est lié à une fréquence caractéristique. La réciproque de celle-ci est une mesure du temps de relaxation du réseau de polymère temporairement formé.

L'augmentation de la masse moléculaire permet l'évolution du point d'intersection vers les faibles fréquences. Puisque la longueur des chaînes polymériques augmente, la capacité de relaxation diminue et le point d'intersection tend vers les faibles fréquences. De plus, pour une masse moléculaire donnée (à des fréquences importantes) $\left(G^{\prime}\right)$ et $\left(\mathrm{G}^{\prime \prime}\right)$ se croisent à des faibles concentrations de polymère. $\mathrm{A}$ des concentrations importantes, la relaxation devient de plus en plus difficile (cela est dû à l'état des segments), le point d'intersection, $G^{\prime}(\omega)=G^{\prime \prime}(\omega)$, se décale alors vers les faibles fréquences.

Ces résultats concordent avec les résultats des courbes d'écoulement et l'application du modèle de Cross (Figure 1c) à travers lequel une diminution du cisaillement caractéristique $(1 / \lambda)$ a été enregistrée (augmentation $\mathrm{du}$ temps de relaxation) (Tableau 2).

Finalement, en se basant sur les expériences d'écoulement, le comportement macroscopique de nos solutions de PEO peut découler de la formation trois réseaux dimensionnels. A des fortes concentrations de polymère, les interactions locales polymèrepolymère (enchevêtrement) sont les principaux facteurs responsables $\mathrm{du}$ comportement rhéologique des solutions de PEO. Ces enchevêtrements sont simultanément ou progressivement détruits et reformés lorsque le cisaillement varie. De ce fait, la concentration critique $\left(c^{* *}\right)$ qui délimite deux états différents de la solution de polymère (les régimes semi-dilué enchevêtré et solution concentrée), peut être considérée comme la concentration critique à laquelle les propriétés viscoélastiques apparaissent dans les solutions de polymère.

On observe aussi un comportement rhéofluidifiant. Celui-ci s'accentue avec la concentration de polymère et est plus important pour la masse moléculaire la plus élevée (Figure 1d).

Ce comportement typique aux solutions de polymères est parfaitement décrit par le modèle de Cross ( Benchabane et al., 2007 ; Ebagninin et al., 2007 ; Ortiz et al., 1994). 
Ces comportements rhéologiques des mélanges bentonite-PEO sont conformes à ceux trouvés par plusieurs auteurs (Alemdar et al., 2005b ; Gungor et Ece, 1999 ; Isci et al., 2004 ; Liu et al., 1996 ; Rossi et al., 2003b, 2002 ,1999, 1997 ; Tunc et Duman, 2008 ; Zaman, 2000).

Dans un système "eau-bentonitepolymères", les chaînes polymériques sont adsorbées sur les particules argileuses par des interactions de types: Van Der Waals, électrostatiques, hydrophobes ou des liaisons hydrogène. Cela conduit soit à un système stérique stable, soit à une floculation (Breen, 1999). Dans le cas des macromolécules neutres, comme le PEO, la fixation à la surface argileuse serait majoritairement due à la création de liaisons hydrogène. D'autres liaisons de type Van der Waals sont également envisagées (Luckham et Rossi, 1999). Dans ce cas, les molécules de polymère peuvent s'adsorber simultanément sur deux ou plusieurs surfaces de particules. Ce phénomène s'accentue avec la concentration et, surtout, avec la masse moléculaire où la longueur des chaînes de polymère permet l'adsorption sur plus d'une particule. Cela forme ce que l'on appelle « une floculation par pont de polymère », causant une augmentation des propriétés rhéologiques du système colloïdal (Isci et al., 2004 ; Luckham et Rossi, 1999).

En outre, on a observé pour la caractérisation des solutions de PEO que, pour les masses moléculaires importantes $\left(4.10^{5}\right.$, $10^{6}$ et $\left.4.10^{6} \mathrm{~g} . \mathrm{mol}^{-1}\right)$, les courbes d'écoulement présentent un caractère nonNewtonien que pour les faibles masses moléculaires $\left(10^{5}\right.$ g.mol $\left.{ }^{-1}\right)$, les solutions sont essentiellement quasi-Newtoniennes.

De ce fait, pour le PEO de masses moléculaires élevées $\left(410^{5}, 10^{6}\right.$ et $4.10^{6}$ g.mol $\left.{ }^{-1}\right)$, l'ajout du PEO à de faibles concentrations (moins de $0,1 \%$ ) provoque une forte augmentation de la viscosité, de la contrainte seuil et des propriétés viscoélastiques de la suspension de base. La longueur des chaînes polymériques créent « une floculation par pont de polymère » où le polymère est adsorbé sur une ou plus particules argileuses.

En revanche, lorsque la chaîne moléculaire n'est pas suffisamment longue, il se crée un effet stérique (Isci et al., 2004 ; Tunc et Duman, 2008 ; Zaman, 2000 ; Zaman et Delorme, 2002). Cela explique la diminution des paramètres rhéologiques observée après l'ajout du PEO de faible masse moléculaire $\left(10^{5} \mathrm{~g} \cdot \mathrm{mol}^{-1}\right)$.

Une étude détaillée de la microstructure (étude du $\mathrm{pH}$, du potentiel zêta, de la granulométrie, d'analyse d'image d'agrégats et de diffraction aux rayons $\mathrm{X}$ ) des mélanges pourra permettre de bien appréhender la relation microstructurale entre les chaînes de polymères et les particules de bentonite.

\section{Conclusion}

Cette étude a permis de comprendre d'une part le comportement rhéologique des solutions de polyéthylène qui serait dû au processus d'enchevêtrement et de désenchevêtrement des chaînes moléculaires. D'autre part de connaître l'effet de l'addition du polyéthylène oxyde (PEO), polymère non ionique de différents masses moléculaires, sur les propriétés rhéologiques d'une suspension de bentonite de base (5\%). Des mesures rhéologiques en écoulement et en régime dynamique ont permis d'établir que le PEO de masses moléculaire élevé $\left(4 \cdot 10^{6} \mathrm{~g} \cdot \mathrm{mol}^{-1}\right)$, et à des faibles concentrations (moins de $0,1 \%$ ) provoque une forte augmentation de la viscosité, de la contrainte seuil et des propriétés viscoélastiques de la suspension de base. En revanche, l'ajout du PEO de faible masse moléculaire entraîne une diminution des paramètres rhéologiques.

Cette réponse rhéologique, fortement viscoélastique, est attribuée à la formation d'une structure en réseau, correspondant à des ponts de polymère entre les particules d'argile.

\section{REFERENCES}

Alemdar A, Güngör N, Ece OI, Atici O. 2005a. The rheological properties and characterization of bentonite dispersions 
in the presence of non-ionic polymer PEG. J. Mater. Sci., 40(1): 171-177.

Alemdar A, Oztekin N, Gungor N, Ece OI, Erim FB. 2005b. Effects of polyethyleneimine adsorption on the rheological properties of purified bentonite suspensions. Colloids and Surfaces A: Phys. Engi. Asp., 252(23):95-98.

Benchabane A, Bekkour K. 2006. Effects of anionic additives on the rheological behavior of aqueous calcium montmorillonite suspensions. Rheol. Acta, 45(4): 425-434.

Benchabane A, Ebagninin KW, Benyounes K, Bekkour K. 2007. Concentrations critiques dans les solutions de polymère. Application aux solutions aqueuses de PEO et de CMC. GFR 2007, ClermontFerrand, 10-12 octobre. p 271-274.

Breen C. 1999. The characterisation and use of polycation-exchanged bentonites. Appl. Clay Sci., 15(1-2): 187-219.

Briscoe B, Luckham P, Zhu S. 1998. Rheological properties of poly(ethylene oxide) aqueous solutions. J. Appl. Poly. Sci., 70(3): 419-429.

Briscoe B, Luckham P, Zhu S. 1996. Rheological Study of Poly(ethylene oxide) in Aqueous Salt Solutions at High Temperature and Pressure. Macromolecules, 29(19): 6208-6211.

Clasen C, Kulicke WM. 2001. Determination of viscoelastic and rheo-optical material functions of water-soluble cellulose derivatives. Prog. Poly. Sci., 26(9): 18391919.

Durán JDG, Ramos-Tejada MM, Arroyo FJ, Gonzalez-Caballero F. 2000. Rheological and electrokinetic properties of sodium montmorillonite suspensions. J. Coll. Interf. Sci., 229(1): 107-117.

Ebagninin KW, Benchabane A, Bekkour K. 2006. L'empreinte "pont de polymère" sur le comportement rhéologique des mélanges bentonite-PEO. GFR 2006, Cherbourg, 18-20 octobre. p 271-274.

Ebagninin KW, Benchabane A, Bekkour K. 2009. Rheological characterization of poly(ethylene oxide) solutions of different molecular weights. Journal of Colloid and Interface Science, 336(1): 360-367.

Gauri V, Koelling KW. 1997. Extensional rheology of concentrated poly(ethylene oxide) solutions. Rheol. Acta, 36(5): 555567.

Gungor N, Ece OI. 1999. Effect of the adsorption of non-ionic polymer poly(vinyl)pyrolidone on the rheological properties of Na-activated bentonite. Mater. Lett., 39(1): 1-5.

Habas J-P, Pavie E, Lapp A, Peyrelasse J. 2008. Non-linear viscoelastic properties of ordered phases of a poly(ethylene oxide)-poly(propylene oxide) triblock copolymer. Rheol. Acta, 47(7): 765-776.

Isci S, Gunister E, Ece OI, Gungor N. 2004. The modification of rheologic properties of clays with PVA effect. Mater. Lett., 58(12-13): 1975-1978.

Kalashnikov VN. 1994. Shear-rate dependent viscosity of dilute polymer solutions. $J$. Rheol., 38(5): 1385-1403.

Kang K, Lee LJ, Koelling KW. 2005. High shear microfluidics and its application in rheological measurement. Exper. Fluids, 38(2): 222-232.

Liu SF, Legrand V, Gourmand M, Lafuma F, Audebert R. 1996. General phase and rheological behavior of silica/peo/water systems. Colloids and Surfaces A: Physic. Engin. Aspects, 111(1-2):139-145.

Luckham PF, Rossi S. 1999. The colloidal and rheological properties of bentonite suspensions. Adva. Coll. Interf. Sci., 82(1-3): 43-92.

Moura MJ, xe, Figueiredo MM, Gil MH. 2007. Rheological Study of Genipin Cross-Linked Chitosan Hydrogels. Biomacromolecules, 8(12): 3823-3829.

Niedzwiedz K, Wischnewski A, PyckhoutHintzen W, Allgaier J, Richter D, Faraone A. 2008. Chain Dynamics and Viscoelastic Properties of Poly(ethylene oxide). Macromolecules, 41(13): 48664872 . 
Ortiz M, De Kee D, Carreau PJ. 1994. Rheology of concentrated poly(ethylene oxide) solutions. J. Rheol., 38(3): 519539.

Powell RL, Schwarz WH. 1975. Rheological Properties of Aqueous Poly(ethylene Oxide) Solutions in Parallel Superposed Flows. J. Rheol., 19(4): 617-643.

Powell RL, Schwarz, WH. 1979. Nonlinear Dynamic Viscoelasticity. J. Rheol., 23(3): 323-352.

Rossi S, Luckham PF, Green N, Cosgrove T. 2003a. NMR solvent relaxation studies of Na+-montmorillonite clay suspensions containing non-ionic polymers. Colloids and Surfaces A: Physic. Engin. Aspects, 215(1-3): 11-24.

Rossi S, Luckham PF, Tadros TF. 2002. Influence of non-ionic polymers on the rheological behaviour of $\mathrm{Na}+-$ montmorillonite clay suspensions--I Nonylphenol-polypropylene oxidepolyethylene oxide copolymers. Colloids and Surfaces A: Physic. Engin. Aspects, 201(1-3): 85-100.

Rossi S, Luckham PF, Tadros TF. 2003b. Influence of non-ionic polymers on the rheological behaviour of $\mathrm{Na}+$ montmorillonite clay suspensions. Part II. Homopolymer ethyleneoxide and polypropylene oxide-polyethylene oxide ABA copolymers. Colloids and Surfaces A: Physic. Engin. Aspects, 215(1-3): 110 .

Rossi S, Luckham PF, Zhu S, Briscoe BJ. 1997. Influence of low molecular weight polymers on the rheology of bentonite suspensions. Revue de l'Institut Français du Pétrole, 52(2): 199-206.
Rossi S, Luckham PF, Zhu S, B.J. Briscoe. 1999. High-pressure/High-Temperature Rheology of Na-Montmorollonite Clay Q Suspensions. Society of Petroleum Engineers SPE 50725:1-14.

Tunc S, Duman O. 2008. The effect of different molecular weight of poly(ethylene glycol) on the electrokinetic and rheological properties of Na-bentonite suspensions. Colloids and Surfaces A: Physic. Engin. Aspects, 317(1-3): 93-99.

Zaman AA. 2000. Effect of polyethylene oxide on the viscosity of dispersions of charged silica particles: interplay between rheology, adsorption, and surface charge. Colloid \& Polymer Sci., 278(12): 11871197.

Zaman AA, Delorme N. 2002. Effect of polymer bridging on rheological properties of dispersions of charged silica particles in the presence of lowmolecular-weight physically adsorbed poly(ethylene oxide). Rheol. Acta, 41(5): 408-417.

Coussot P, Gossiord JL. 2001. Comprendre la Rhéologie de la Circulation du Sang à la Prise du Béton. EDP Sciences ; 221.

Benchabane A. 2006. Etude du comportement rhéologique de mélanges argiles polymères. Effets de l'ajout de polymères. Thèse unique, France, Strasbourg ; p169. 